\title{
ESSENTIAL RADICAL SUPPLEMENTED MODULES
}

\author{
CELIL NEBIYEV
}

Received 3 February, 2020

\begin{abstract}
In this work, (amply) essential radical supplemented modules are defined and some properties of these modules are investigated. Let $M$ be an $R$-module and $M=M_{1}+M_{2}+\cdots+$ $M_{n}$. If $M_{i}$ is essential radical supplemented for every $i=1,2, \ldots, n$, then $M$ is also essential radical supplemented. It is proved that every factor module and every homomorphic image of an essential radical supplemented module are essential radical supplemented. Let $M$ be an essential radical supplemented $R$-module. Then every finitely $M$-generated $R$-module is essential radical supplemented.
\end{abstract}

2010 Mathematics Subject Classification: 16D10; 16D70

Keywords: small submodules, essential submodules, radical, generalized (radical) supplemented modules

\section{INTRODUCTION}

Throughout this paper all rings will be associative with identity and all modules will be unital left modules.

Let $M$ be an $R$-module and $N \leq M$. If $L=M$ for every submodule $L$ of $M$ such that $M=N+L$, then $N$ is called a small (or superfluous) submodule of $M$ and denoted by $N \ll M$. A submodule $N$ of an $R$-module $M$ is called an essential submodule of $M$ and denoted by $N \unlhd M$ in case $K \cap N \neq 0$ for every submodule $K \neq 0$, or equivalently, $N \cap L=0$ for $L \leq M$ implies that $L=0$. Let $M$ be an $R$-module and $K$ be a submodule of $M . K$ is called a generalized small (briefly, $g$-small) submodule of $M$ if for every essential submodule $T$ of $M$ with the property $M=K+T$ implies that $T=M$, then we write $K \ll_{g} M$. It is clear that every small submodule is a generalized small submodule but the converse is not true generally. Let $M$ be an $R$-module and $U, V \leq M$. If $M=U+V$ and $V$ is minimal with respect to this property, or equivalently, $M=U+V$ and $U \cap V \ll V$, then $V$ is called a supplement of $U$ in $M$. $M$ is called a supplemented module if every submodule of $M$ has a supplement in $M$. Let $M$ be an $R$-module and $U \leq M$. If for every $V \leq M$ such that $M=U+V, U$ has a supplement $V^{\prime}$ with $V^{\prime} \leq V$, we say $U$ has ample supplements in $M$. If every submodule of $M$ has ample supplements in $M$, then $M$ is called an amply supplemented module. If every essential submodule of $M$ has a supplement in $M$, then $M$ is called 
an essential supplemented (or briefly, e-supplemented) module. If every essential submodules of $M$ has ample supplements in $M$, then $M$ is called an amply essential supplemented (or briefly, amply e-supplemented) module. Let $M$ be an $R$-module and $U, V \leq M$. If $M=U+V$ and $M=U+T$ with $T \unlhd V$ implies that $T=V$, or equivalently, $M=U+V$ and $U \cap V \ll_{g} V$, then $V$ is called a $g$-supplement of $U$ in $M$. $M$ is said to be $g$-supplemented if every submodule of $M$ has a g-supplement in $M$. The intersection of all maximal submodules of an $R$-module $M$ is called the radical of $M$ and denoted by $\operatorname{Rad} M$. If $M$ have no maximal submodules, then we denote $\operatorname{Rad} M=M$. The intersection of essential maximal submodules of an $R$-module $M$ is called the generalized radical of $M$ and denoted by $\operatorname{Rad}_{g} M$. If $M$ have no essential maximal submodules, then we denote $\operatorname{Rad}_{g} M=M$. Let $M$ be an $R$-module and $U, V \leq M$. If $M=U+V$ and $U \cap V \leq \operatorname{Rad} V$, then $V$ is called a generalized (radical) supplement (or briefly, Rad-supplement) of $U$ in $M . M$ is called a generalized (radical) supplemented (or briefly, Rad-supplemented) module if every submodule of $M$ has a Rad-supplement in $M$. Let $M$ be an $R$-module and $U \leq M$. If for every $V \leq M$ such that $M=U+V, U$ has a Rad-supplement $V^{\prime}$ with $V^{\prime} \leq V$, we say $U$ has ample Rad-supplements in $M$. If every submodule of $M$ has ample Rad-supplements in $M$, then $M$ is called an amply generalized (radical) supplemented (or briefly, amply Rad-supplemented) module. Let $M$ be an $R$-module. We say submodules $X$ and $Y$ of $M$ are $\beta^{*}$ equivalent, $X \beta^{*} Y$, if and only if $Y+K=M$ for every $K \leq M$ such that $X+K=M$ and $X+T=M$ for every $T \leq M$ such that $Y+T=M$. Let $M$ be an $R$-module $X \leq Y \leq M$. If $Y / X \ll M / X$, then we say $Y$ lies above $X$ in $M$.

More information about (amply) supplemented modules are in [3, 9, 10] and [11]. More information about (amply) essential supplemented modules are in [5, 6]. More results about g-small submodules and g-supplemented modules are in [4,7]. The definitions of (amply) generalized supplemented modules and some properties of them are in $[8,10]$. Some properties of (amply) generalized supplemented modules are also in [2]. The definition of $\beta^{*}$ equivalence relation and some properties of this relation are in [1].

In this paper, we define (amply) essential radical supplemented modules and investigate some properties about these modules. We constitute relationships between essential radical supplemented modules and amply essential radical supplemented modules by Proposition 3 and Proposition 4. We also constitute relationships between essential radical supplemented modules and $\pi$-projective modules by Lemma 12 . We give two examples for essential radical supplemented modules separating with essential supplemented modules at the end of this paper.

Lemma 1. Let $M$ be an R-module and $K \leq N \leq M$. If $K$ is a generalized small submodule of $N$, then $K$ is a generalized small submodule in submodules of $M$ which contain $N$.

Proof. See [4, Lemma 1 (2)]. 
Lemma 2. Let $M$ be an R-module. Then $\operatorname{Rad}_{g} M=\sum_{L \ll_{g} M} L$.

Proof. See [4, Lemma 5 and Corollary 5].

Lemma 3. Let $V$ be a Rad-supplement of $U$ in $M$. Then $\operatorname{Rad} V=V \cap \operatorname{Rad} M$.

Proof. Let $T$ be any maximal submodule of $V$. Since

$$
M /(U+T)=(U+T+V) /(U+T) \cong V /(U \cap V+T)=V / T,
$$

then $U+T$ is a maximal submodule of $M$. Hence $\operatorname{Rad} M \leq U+T$ and $V \cap \operatorname{Rad} M \leq$ $U \cap V+T=T$. Thus $V \cap \operatorname{Rad} M \leq \operatorname{Rad} V$ and since $\operatorname{Rad} V \leq V \cap \operatorname{Rad} M, \operatorname{Rad} V=$ $V \cap \operatorname{Rad} M$.

\section{ESSENTIAL RADICAL SUPPLEMENTED MODULES}

Definition 1. Let $M$ be an $R$-module. If every essential submodule of $M$ has a Rad-supplement in $M$, then $M$ is called an essential radical supplemented (or briefly, e-Rad-supplemented) module.

Clearly we see that every essential supplemented module is essential radical supplemented. But the converse is not true in general. (See Examples 1 and 2).

Definition 2. Let $M$ be an $R$-module and $X \leq M$. If $X$ is a Rad-supplement of an essential submodule in $M$, then $X$ is called an essential radical supplement (or briefly, e-Rad-supplement) submodule in $M$.

Lemma 4. Let $M$ be an $R$-module, $V$ be an e-Rad-supplement in $M$ and $x \in V$. Then $R x \ll_{g} M$ if and only if $R x \ll_{g} V$.

Proof. ( $\Longrightarrow)$ Let $R x \ll_{g} M$. Since $V$ is an e-Rad-supplement in $M$, there exists $U \unlhd M$ such that $V$ is a Rad-supplement of $U$ in $M$. Let $R x+T=V$ with $T \unlhd V$. Then $M=U+V=U+T+R x$, and since $R x \ll_{g} M$ and $(U+T) \unlhd M, U+T=M$. Let $x=u+t$ with $u \in U$ and $t \in T$. Since $x, t \in V$, then $u=x-t \in V$. Then $V=$ $R x+T \leq R u+R t+T=R u+T \leq V$ and $R u+T=V$. Since $u \in U \cap V \leq \operatorname{Rad} V$, then $R u \ll V$ and $T=V$. Hence $R x \ll_{g} V$.

$(\Longleftarrow)$ Clear from Lemma 1 .

Corollary 1. Let $M$ be an R-module and $V$ be an e-Rad-supplement in $M$. Then $\operatorname{Rad}_{g} V=V \cap \operatorname{Rad}_{g} M$.

Proof. Let $x \in \operatorname{Rad}_{g} V$. Here $R x \ll_{g} V$ and by Lemma $1, R x \ll_{g} M$. Then by Lemma 2, $R x \leq \operatorname{Rad}_{g} M$ and $x \in V \cap \operatorname{Rad}_{g} M$.

Let $y \in V \cap \operatorname{Rad}_{g} M$. Then $y \in V$ and $R y \ll_{g} M$. By Lemma 4, $R y \ll_{g} V$. By Lemma 2, $R y \leq \operatorname{Rad}_{g} V$ and $y \in \operatorname{Rad}_{g} V$.

Hence $\operatorname{Rad}_{g} V=V \cap \operatorname{Rad}_{g} M$.

Proposition 1. Let $M$ be an essential radical supplemented module. Then $M / \operatorname{Rad} M$ have no proper essential submodules. 
Proof. Let $\frac{K}{\operatorname{Rad} M}$ be any essential submodule of $\frac{M}{\operatorname{Rad} M}$. Since $\frac{K}{\operatorname{Rad} M} \unlhd \frac{M}{\operatorname{Rad} M}, K \unlhd M$ and since $M$ is essential radical supplemented, $K$ has a Rad-supplement $V$ in $M$. Then $M=K+V$ and $K \cap V \leq \operatorname{Rad} V$. Since $M=K+V, \frac{M}{\operatorname{Rad} M}=\frac{K}{\operatorname{Rad} M}+\frac{V+\operatorname{Rad} M}{\operatorname{Rad} M}$. Since $K \cap V \leq \operatorname{Rad} M$, then $\frac{K}{\operatorname{Rad} M} \cap \frac{V+\operatorname{Rad} M}{\operatorname{Rad} M}=\frac{K \cap V+\operatorname{Rad} M}{\operatorname{Rad} M}=0$ and $\frac{M}{\operatorname{Rad} M}=\frac{K}{\operatorname{Rad} M} \oplus \frac{V+\operatorname{Rad} M}{\operatorname{Rad} M}$. Since $\frac{M}{\operatorname{Rad} M}=\frac{K}{\operatorname{Rad} M} \oplus \frac{V+\operatorname{Rad} M}{\operatorname{Rad} M}$ and $\frac{K}{\operatorname{Rad} M} \unlhd \frac{M}{\operatorname{Rad} M}, \frac{K}{\operatorname{Rad} M}=\frac{M}{\operatorname{Rad} M}$. Hence $\frac{M}{\operatorname{Rad} M}$ have no proper essential submodules.

Lemma 5. Let $M$ be an $R$-module, $U$ be an essential submodule of $M$ and $M_{1} \leq M$. If $M_{1}$ is e-Rad-supplemented and $U+M_{1}$ has a Rad-supplement in $M$, then $U$ has a Rad-supplement in $M$.

Proof. Let $X$ be a Rad-supplement of $U+M_{1}$ in $M$. Then $M=U+M_{1}+X$ and $X \cap\left(U+M_{1}\right) \leq \operatorname{Rad} X$. Since $U \unlhd M,(U+X) \unlhd M$ and $(U+X) \cap M_{1} \unlhd M_{1}$. Since $M_{1}$ is e-Rad-supplemented, $(U+X) \cap M_{1}$ has a Rad-supplement $Y$ in $M_{1}$. This case $M_{1}=(U+X) \cap M_{1}+Y$ and $(U+X) \cap Y=(U+X) \cap M_{1} \cap Y \leq \operatorname{Rad} Y$. Then $M=U+M_{1}+X=U+X+(U+X) \cap M_{1}+Y=U+X+Y$ and $U \cap(X+Y) \leq$ $(U+X) \cap Y+(U+Y) \cap X \leq\left(U+M_{1}\right) \cap X+(U+X) \cap Y \leq \operatorname{Rad} X+\operatorname{Rad} Y \leq$ $\operatorname{Rad}(X+Y)$. Hence $X+Y$ is a $\operatorname{Rad}$-supplement of $U$ in $M$.

Corollary 2. Let $M$ be an $R$-module, $U$ be an essential submodule of $M$ and $M_{i} \leq$ $M$ for every $i=1,2, \ldots, n$. If $M_{i}$ is e-Rad-supplemented for every $i=1,2, \ldots, n$ and $U+M_{1}+M_{2}+\cdots+M_{n}$ has a Rad-supplement in $M$, then $U$ has a Rad-supplement in $M$.

Proof. Clear from Lemma 5.

Lemma 6. Let $M=M_{1}+M_{2}$. If $M_{1}$ and $M_{2}$ are e-Rad-supplemented, then $M$ is also e-Rad-supplemented.

Proof. Let $U \unlhd M$. Then 0 is a Rad-supplement of $U+M_{1}+M_{2}$ in $M$. Since $M_{2}$ is e-Rad-supplemented and $\left(U+M_{1}\right) \unlhd M$, by Lemma $5, U+M_{1}$ has a Rad-supplement in $M$. Since $M_{1}$ is e-Rad-supplemented and $U \unlhd M$, by Lemma $5, U$ has a Rad-supplement in $M$. Hence $M$ is e-Rad-supplemented.

Corollary 3. Let $M=M_{1}+M_{2}+\cdots+M_{n}$. If $M_{i}$ is e-Rad-supplemented for each $i=1,2, \ldots, n$, then $M$ is also e-Rad-supplemented.

Proof. Clear from Lemma 6.

Lemma 7. Every factor module of an e-Rad-supplemented module is e-Rad-supplemented.

Proof. Let $M$ be an e-Rad-supplemented $R$-module and $\frac{M}{K}$ be any factor module of $M$. Let $\frac{U}{K} \unlhd \frac{M}{K}$. Then $U \unlhd M$ and since $M$ is e-Rad-supplemented, $U$ has a Rad-supplement $V$ in $M$. Since $K \leq U$, by the proof of [8, Proposition 2.6(1)], $\frac{V+K}{K}$ is a Rad-supplement of $\frac{U}{K}$ in $\frac{M}{K}$. Hence $\frac{M}{K}$ is e-Rad-supplemented. 
Corollary 4. Every homomorphic image of an e-Rad-supplemented module is e-Rad-supplemented.

Proof. Clear from Lemma 7.

Lemma 8. Let $M$ be an e-Rad-supplemented R-module. Then every finitely $M$-generated R-module is e-Rad-supplemented.

Proof. Let $N$ be a finitely $M$-generated $R$-module. Then there exist a finite index set $\Lambda$ and an $R$-module epimorphism $f: M^{(\Lambda)} \longrightarrow N$. Since $M$ is e-Rad-supplemented, by Corollary 3, $M^{(\Lambda)}$ is e-Rad-supplemented. Then by Corollary 4, $N$ is e-Rad-supplemented.

Proposition 2. Let $R$ be a ring. Then ${ }_{R} R$ is essential radical supplemented if and only if every finitely generated $R$-module is essential radical supplemented.

Proof. Clear from Lemma 8.

Lemma 9. Let $M$ be an $R$-module. If every essential submodule of $M$ is $\beta^{*}$ equivalent to an e-Rad-supplement submodule in $M$, then $M$ is essential radical supplemented.

Proof. Let $U$ be an essential submodule of $M$. By hypothesis there exists an e-Rad-supplement submodule $X$ in $M$ such that $U \beta^{*} X$. Since $X$ is an e-Rad-supplement submodule in $M$, there exists an essential submodule $Y$ of $M$ such that $X$ is a Rad-supplement of $Y$ in $M$. This case $M=X+Y$ and $X \cap Y \leq \operatorname{Rad} X$. Since $Y \unlhd M$, by hypothesis, there exists an e-Rad-supplement submodule $V$ in $M$ such that $Y \beta^{*} V$. Since $U \beta^{*} X$ and $M=X+Y$, then $M=U+Y$ and since $Y \beta^{*} V, M=U+V$. Let $x \in U \cap V$ and $R x+T=M$ with $T \leq M$. Then $U \cap V+T=M$ and since $M=U+V$, $M=U+V \cap T=X+V \cap T$. Since $M=V+T=X+V \cap T, M=V+X \cap T$. Then by $Y \beta^{*} V, M=Y+X \cap T$. Since $M=X+T=Y+X \cap T, M=X \cap Y+T$. Let $x=y+t$, with $y \in X \cap Y$ and $t \in T$. Since $R x+T=M, R y+T=M$ also holds. By $y \in X \cap Y \leq \operatorname{Rad} X \leq \operatorname{Rad} M, R y \ll M$ and since $R y+T=M, T=M$. Hence $R x \ll M$ and $x \in \operatorname{Rad} M$. Since $V$ is a Rad-supplement in $M$, then by Lemma 3, $V \cap \operatorname{Rad} M=$ $\operatorname{Rad} V$. Since $x \in V$ and $x \in \operatorname{Rad} M, x \in V \cap \operatorname{Rad} M=\operatorname{Rad} V$ and $U \cap V \leq \operatorname{Rad} V$. Hence $V$ is a Rad-supplement of $U$ in $M$ and $M$ is essential radical supplemented.

Corollary 5. Let $M$ be an R-module. If every essential submodule of $M$ lies above an e-Rad-supplement submodule in $M$, then $M$ is essential radical supplemented.

Proof. Clear from Lemma 9.

\section{AMPLy ESSENTIAL RADICAL SUPPLEMENTED MODULES}

Definition 3. Let $M$ be an $R$-module. If every essential submodule has ample Rad-supplements in $M$, then $M$ is called an amply essential radical supplemented (or briefly, amply e-Rad-supplemented) module. 
Lemma 10. Let $M$ be an amply e-Rad-supplemented module. Then every factor module of $M$ is amply e-Rad-supplemented.

Proof. Let $M / K$ be any factor module of $M, U / K \unlhd M / K$ and $U / K+V / K=$ $M / K$ with $V / K \leq M / K$. Since $U / K \unlhd M / K, U \unlhd M$. Since $U / K+V / K=M / K$, $U+V=M$. Because $M$ is amply e-Rad-supplemented, $U$ has a Rad-supplement $V^{\prime}$ in $M$ with $V^{\prime} \leq V$. By the proof of [8, Proposition 2.6(1)], $\frac{V^{\prime}+K}{K}$ is a Rad-supplement of $\frac{U}{K}$ in $\frac{M}{K}$. In addition to this, $\frac{V^{\prime}+K}{K} \leq \frac{V}{K}$. Hence $M / K$ is amply e-Rad-supplemented.

Corollary 6. Let $M$ be an amply e-Rad-supplemented module. Then every homomorphic image of $M$ is amply e-Rad-supplemented.

Proof. Clear from Lemma 10.

Lemma 11. Let $M$ be an R-module. If every submodule of $M$ is e-Rad-supplemented, then $M$ is amply e-Rad-supplemented.

Proof. Let $M=U+V$ with $U \unlhd M$ and $V \leq M$. By hypothesis, $V$ is e-Rad-supplemented. Since $U \unlhd M, U \cap V \unlhd V$. Since $V$ is e-Rad-supplemented, $U \cap V$ has a Rad-supplement $K$ in $V$. Here $U \cap V+K=V$ and $U \cap K=U \cap V \cap K \leq \operatorname{Rad} K$. Then $M=U+V=U+U \cap V+K=U+K$ and $U \cap K \leq \operatorname{Rad} K$. Hence $M$ is amply e-Rad-supplemented.

Proposition 3. Let $R$ be any ring. Then every $R$-module is e-Rad-supplemented if and only if every $R$-module is amply e-Rad-supplemented.

Proof. ( $\Longrightarrow)$ Let $M$ be any $R$-module. Since every $R$-module is e-Rad-supplemented, every submodule of $M$ is e-Rad-supplemented. Then by Lemma 11, $M$ is amply e-Rad-supplemented.

$(\Longleftarrow)$ Clear.

Lemma 12. Let $M$ be a $\pi$-projective and e-Rad-supplemented R-module. Then $M$ is amply e-Rad-supplemented.

Proof. Let $U \unlhd M, M=U+V$ and $X$ be a Rad-supplement of $U$ in $M$. Since $M$ is $\pi$-projective and $M=U+V$, there exists an $R$-module homomorphism $f: M \rightarrow M$ such that $\operatorname{Im} f \subset V$ and $\operatorname{Im}(1-f) \subset U$. So, we have $M=f(M)+(1-f)(M)=$ $f(U)+f(X)+U=U+f(X)$. Suppose that $a \in U \cap f(X)$. Since $a \in f(X)$, then there exists $x \in X$ such that $a=f(x)$. Since $a=f(x)=f(x)-x+x=x-(1-f)(x)$ and $(1-f)(x) \in U$, we have $x=a+(1-f)(x) \in U$. Thus $x \in U \cap X$ and so, $a=f(x) \in f(U \cap X)$. Therefore we have $U \cap f(X) \leq f(U \cap X) \leq f(\operatorname{Rad} X) \leq$ $\operatorname{Rad} f(X)$. This means that $f(X)$ is a $\operatorname{Rad}$-supplement of $U$ in $M$. Moreover, $f(X) \subset$ $V$. Therefore $M$ is amply e-Rad-supplemented.

Corollary 7. If $M$ is a projective and e-Rad-supplemented module, then $M$ is an amply e-Rad-supplemented module. 
Proof. Clear from Lemma 12.

Proposition 4. Let $R$ be a ring. The following assertions are equivalent.

(i) ${ }_{R} R$ is e-Rad-supplemented

(ii) ${ }_{R} R$ is amply e-Rad-supplemented.

(iii) Every finitely generated R-module is e-Rad-supplemented.

(iv) Every finitely generated R-module is amply e-Rad-supplemented.

Proof. $(i) \Longleftrightarrow($ ii $)$ Clear from Corollary 7 , since ${ }_{R} R$ is projective.

$(i) \Longrightarrow$ (iii) Clear from Lemma 8.

(iii) $\Longrightarrow($ iv $)$ Let $M$ be a finitely generated $R$-module. Then there exist a finite index set $\Lambda$ and an $R$-module epimorphism $f: R^{(\Lambda)} \longrightarrow M$. Since every finitely generated $R$-module is e-Rad-supplemented, $R^{(\Lambda)}$ is e-Rad-supplemented. Since ${ }_{R} R$ is projective, $R^{(\Lambda)}$ is also projective. Then by Corollary $7, R^{(\Lambda)}$ is amply e-Rad-supplemented. Since $f: R^{(\Lambda)} \longrightarrow M$ is an $R$-module epimorphism, by Corollary 6, $M$ is also amply e-Rad-supplemented.

(iv) $\Longrightarrow($ i) Clear.

Example 1. Consider the $\mathbb{Z}$-module $\mathbb{Q}$. Since $\operatorname{Rad} \mathbb{Q}=\mathbb{Q}, \mathbb{Z} \mathbb{Q}$ is essential radical supplemented. But, since $\mathbb{Z} \mathbb{Q}$ is not supplemented and every nonzero submodule of $\mathbb{Z} \mathbb{Q}$ is essential in $\mathbb{Z} \mathbb{Q}, \mathbb{Q} \mathbb{Q}$ is not essential supplemented.

Example 2. Consider the $\mathbb{Z}$-module $\mathbb{Q} \oplus \mathbb{Z}$, for a prime $p$. It is easy to check that $\operatorname{Rad}\left(\mathbb{Q} \oplus \mathbb{Z}_{p}\right)=\mathbb{Q} \neq \mathbb{Q} \oplus \mathbb{Z}_{p}$. Since $\mathbb{Q}$ and $\mathbb{Z}_{p}$ are essential radical supplemented, by Lemma $6, \mathbb{Q} \oplus \mathbb{Z}_{p}$ is essential radical supplemented. But $\mathbb{Q} \oplus \mathbb{Z}_{p}$ is not essential supplemented.

\section{REFERENCES}

[1] G. F. Birkenmeier, F. Takil Mutlu, C. Nebiyev, N. Sokmez, and A. Tercan, "Goldie*-supplemented modules," Glasg. Math. J., vol. 52, no. A, pp. 41-52, 2010, doi: 10.1017/S0017089510000212.

[2] E. Büyükaşik and C. Lomp, "On a recent generalization of semiperfect rings," Bull. Aust. Math. Soc., vol. 78, no. 2, pp. 317-325, 2008, doi: 10.1017/S0004972708000774.

[3] J. Clark, C. Lomp, N. Vanaja, and R. Wisbauer, Lifting modules: Supplements and projectivity in module theory, ser. Frontiers in Mathematics. Birkhäuser Verlag, Basel, 2006.

[4] B. Koşar, C. Nebiyev, and N. Sökmez, "g-supplemented modules," Ukrainian Math. J., vol. 67, no. 6, pp. 975-980, 2015, translation of Ukraïn. Mat. Zh. 67 (2015), no. 6, 861-864, doi: 10.1007/s11253-015-1127-8.

[5] C. Nebiyev, H. H. Ökten, and A. Pekin, "Essential supplemented modules," International Journal of Pure and Applied Mathematics, vol. 120, no. 2, pp. 253-257, 2018, doi: 10.12732/ijpam.v120i2.9.

[6] C. Nebiyev, H. H. Ökten, and A. Pekin, "Amply essential supplemented modules," Journal of Scientific Research and Reports, vol. 21, no. 4, pp. 1-4, 2018, doi: 10.9734/JSRR/2018/45651.

[7] N. Sökmez, B. Koşar, and C. Nebiyev, "Genelleştirilmiş küçük alt modüller," in XIII. Ulusal Matematik Sempozyumu. Kayseri: Erciyes Üniversitesi, 2010.

[8] Y. Wang and N. Ding, "Generalized supplemented modules," Taiwanese J. Math., vol. 10, no. 6, pp. 1589-1601, 2006, doi: 10.11650/twjm/1500404577. 
[9] R. Wisbauer, Foundations of module and ring theory, german ed., ser. Algebra, Logic and Applications. Gordon and Breach Science Publishers, Philadelphia, PA, 1991, vol. 3, a handbook for study and research.

[10] W. Xue, "Characterizations of semiperfect and perfect rings," Publ. Mat., vol. 40, no. 1, pp. 115125, 1996, doi: 10.5565/PUBLMAT_40196_08.

[11] H. Zöschinger, "Komplementierte Moduln über Dedekindringen,” J. Algebra, vol. 29, pp. 42-56, 1974, doi: 10.1016/0021-8693(74)90109-4.

Author's address

Celil Nebiyev

Ondokuz Mayıs University, 55270, Kurupelit, Atakum, Samsun, Turkey

E-mail address: cnebiyev@omu.edu.tr 